surface antigens detectable in mixed lymphocyte reactions, and other loci controlling various components of complement and transplantation antigens. Some 20 allelic variants of the $A$ and $B$ antigens are now recognised, and polymorphism of the $\mathrm{C}$ and $\mathrm{D}$ locus antigens is also established.

The First International Symposium on HLA and Disease ${ }^{4}$ was a unique blend of clinicians and experimental scientists, each group with something to learn and to impart in return. Histocompatibility antigens, which until recently were regarded as interesting only to mouse geneticists and some transplantation surgeons, have suddenly become the common property of many biological sciences. We are still exploring the implications of the discovery in mouse and man of specialised genetic mechanisms associated with immune responses and disease.

To the practising clinician the relevance of this collaborative venture is the question, "Will HI.A help me to diagnose or treat my patients more effectively ?" If experiments on mice are any guide, the answers will not be simple. Early studies showed that in mice susceptibility or resistance to disease (in particular some virus infections) was strongly influenced by genes within the MHC. ${ }^{5}$ Later it became clear that not all immune responses were the result of genes within this complex. In some infections or responses additional genes on several other chromosomes were implicated. ${ }^{6}$ Recently, it has become evident that some tissue cells, in particular B lymphocytes and monocytes, carry immune associated antigens comparable with those already described in mice and that these, too, are either closely related or identical to the HLA D antigens. $^{7}$

Many of the HLA associations fall into two broad groups. Firstly, there is a category showing strong association with the B locus antigens. These tend to be diseases with a male predominance, and sometimes there is indirect evidence of bacterial infection. The association of the B27 antigen with ankylosing spondylitis, Reiter's disease, and uveitis are wellestablished examples. Secondly, some diseases originally found to be associated relatively weakly with B locus antigens have now been shown to have a closer association with the $\mathrm{D}$ locus. Often there is a female predominance in these diseases, and in some a latent virus infection is suspected. Examples include multiple sclerosis, which is associated with the DW2 antigen (W indicates a "workshop" nomenclature), gluten sensitive enteropathy and dermatitis herpetiformis (DW3), and very recently rheumatoid arthritis, where an association with the DW4 antigen was reported by four independent groups at the meeting. Among the puzzling features is the preponderance of diseases with a strong hereditary component but an unknown aetiology, such as juvenile diabetes. Furthermore, in mice the strongest associations have been between MHC antigens and the growth of tumours induced by oncogenic RNA viruses, whereas in man HLA associations with malignant disease have been among the weakest described.

Four main hypotheses have been proposed to account for these observations. Firstly the simplest, molecular mimicry hypothesis, suggests that the HLA antigen is cross-reactive with the causative agent.5 Secondly, HLA antigens, being cellsurface structures, might act as convenient receptors for viruses or bacterial products which might then trigger disease. One finding consistent with this theory is the association of BW35 and non-streptococcal (probably viral) glomerulonephritis, reported independently by three groups in Paris. ${ }^{4} \mathrm{~A}$ third mechanism proposed at the meeting by Svejgaard contained elements of both the molecular mimicry and the receptor hypotheses, since it invoked a structural similarity between certain HI.A antigens and, say, hormone receptors. The resultant competitive binding could cause the hormone to be rapidly absorbed on to the wrong receptors.

The fourth proposed mechanism, popular with immunologists, is the immune response gene. This hypothesis envisages a class of molecules distinct from immunoglobulins but capable of interacting specifically with antigen and composed partially or totally of gene products of the MHC.

Immune response genes are known to occur in the mouse MHC, and if they exist in man they may share some of the unusual genetic features of HLA genes. Some A and B antigens of the HLA system occur together more often than would be expected if the genes underwent normal random segregation. ${ }^{8}$ For example, the haplotype $\mathrm{Al}, \mathrm{B} 8$ occurs more frequently in European populations than would be predicted by the product of the separate gene frequencies for $\mathrm{Al}$ and $\mathrm{B} 8$. This phenomenon is known as linkage disequilibrium. Disequilibrium within the MHC linkage is known to occur not only between $A$ and $B$ alleles but also between $B$ and $D$. If the immune response system does indeed have genes in linkage disequilibrium with HLA, and especially D locus genes, this would explain the HLA antigen associations with disease. The strength of such an association would depend in part on the degree of linkage disequilibrium between a particular HLA gene and the appropriate immune response genes, and this linkage might differ in separate racial groups.

Our continuing ignorance of the biological significance of the HLA system should warn us against ill-considered demands for large-scale population screening, at least in the short term. The diagnostic usefulness of HLA typing remains strictly limited and more detailed mapping of "disease associated genes" must be the next goal. The specificities of the immune antigen system and its genetic relation to HLA will be the subject of the 7th International Workshop to be held at Oxford in 1977.

${ }^{1}$ Dausset, J, Acta haematologica, 1958, 20, 156.

${ }^{2}$ Van Rood, J J, and van Leeuwen, A, fournal of Clinical Investigation, 1963, 42, 1382 .

${ }^{3}$ Payne, R, et al, Cold Spring Harbor Symposia of Quantitative Biology, $1964,29,285$.

${ }^{4} H L A$ and Disease, Abstracts. Paris, Editions Inserm, 1976.

5 Snell, G D, Folia Biologica (Praha), 1968, 14, 335.

${ }^{6} \mathrm{McDevitt}, \mathrm{H}$, and Landy, M, Genetic Control of Immune Responsiveness. New York and London, Academic Press, 1972.

${ }^{7}$ Histocompatibility Testing, ed F Kissmeyer-Nielsen. Copenhagen, Munkgaard, 1975.

Bodmer, W F, Nature, 1972, 237, 139

${ }^{9}$ Oliver, R T D, Klouda, P, and Lawler, S, HLA and Disease, Abstracts. Paris, Editions Inserm, 1976.

\section{Contaminated infusion fluids}

So commonplace is the setting up of intravenous infusions that familiarity may breed contempt. Most residents would be frankly sceptical of the suggestion that it is a potentially dangerous procedure which may cause death. The major risk is from infection. Organisms may gain access at the drip site, ${ }^{1}$ especially if there is leakage from the development of phlebitis, and a wide range of pathogens may be responsible for such individual episodes.

We have come to expect that commercial firms will provide guaranteed sterile fluids for infusion, and, indeed, it is most unusual for fluids to be contaminated. Breakdowns may occur in the sterile preparation procedure, however, but their very 
infrequency contributes to the difficulty of recognising and avoiding these episodes. The lapse may result from human or mechanical failure, combinations of which came to light in the last episode in Britain, in the Plymouth area. ${ }^{2}$ Rapidly changing technology, particularly during economic stringency, may also cause problems-and the introduction of plastic infusion packs will not necessarily eliminate the risk. A recent report ${ }^{3}$ from the Centre for Disease Control, Atlanta, of an outbreak of infection occurring in the USA in 1970-71, has provided a useful summary of many points which are required knowledge for all concerned with giving intravenous fluids and for microbiologists responsible for investigating reactions thought to have resulted from their use.

The American outbreak, bigger than anything we have experienced in Britain, followed the nationwide use of the contaminated fluid. To investigate the outbreak 25 hospitals were chosen which yielded 378 cases of septicaemia. One fact that emerged was that hospitals with a team including an infection control nurse continually monitoring nosocomial infection were early to spot that an outbreak was in progress. Other institutions (including some large hospitals) where adequate infection control surveillance was not practised became aware that they had been concerned in the outbreak only in retrospect. Nevertheless, the "epidemic" organism was isolated from only six of 1054 random samples of fluid stocks and the overall attack rate was only 1.4 cases per 1000 hospital patients, so that the occult nature of the outbreak was hardly surprising. A few species only of the genus Enterobacter were implicated-a finding in keeping with previous reports, even from Britain. ${ }^{4}$ They included $E$ agglomerans, until recently regarded as a plant pathogen only. All organisms of the Klebsiella-Enterobacter-Serratia group were found to possess a singular ability to multiply in commercial glucose solutions at room temperature, attaining numbers above $10^{8}$ organisms litre within 24 hours. Other organisms, including staphylococci, Proteus, Pseudomonas, and Escherichia coli, either failed to grow or died. 5

Once organisms able to grow in glucose solutions have been introduced into a drip they can persist despite rapid rates of flow of the infusion and even after the bottle has been changed. ${ }^{6} 7$ For this reason Maki et al recommend that the bottle and giving set "down to but not necessarily including the cannula" should be replaced every 24 hours. This advice may be questioned since thrombosis starts in the cannula, which is the favoured site for bacterial colonisation. If routine replacement is to be recommended it must surely include the cannula, but this does not seem justifiable. Conversely, the further recommendation-that, should the cannula need replacing, the entire administration set, including any halfused bottle, should be discarded-is reasonable, as it often applies to long-standing drips, which are particularly prone to infection.

A point favouring the early detection of an outbreak is to identify precisely Gram-negative rods, the organisms concerned being relatively uncommon isolates from ordinary episodes of septicaemia. Some commercially available biochemical sets, particularly those linked to a diagnostic register, may help in this task since they minimise the element of individual guessing in bacteriological identification. When a strain of $E$ agglomerans isolated during the outbreak was subsequently circulated as part of a laboratory quality control programme, $45 \%$ of 250 laboratories taking part failed to identify it correctly. Investigation of these outbreaks should include preservation of all apparatus for examination and culture, noting batch numbers of fluids, withdrawal of sup- plies, and random bacteriological sampling. The method of adding $\times 10$ strength media to whole bottles of fluid is both practicable and sensitive.

It is difficult to advise those responsible for giving intravenous fluids how to detect contaminated batches in practice, and so avoid these episodes. Holding bottles up even to the strongest light will detect only gross contamination-and also tends not actually to be done, especially at night. The most vital point is that the first person to notice any untoward reaction should stop the infusion at the first sign. Once a doctor has been called to confirm this decision (to avoid discontinuing valuable drips which have taken time and skill to put up), the drip should be taken down and the entire apparatus submitted (without contaminating it) for bacteriological culture. In these circumstances antibiotic treatment alone is prone to fail. In the reported outbreak, 17 of 19 patients in whom the administration of the fluid was continued had positive blood cultures 24 hours or more after starting gentamicin therapy.

The most important aspect is to be aware that such outbreaks are possible. With this in view everyone should read this informative report. Overall the episode was thought to have contributed to the deaths of 40 patients. Similar incidents have already occurred in Britain, and undoubtedly they will occur again. When they do, how long will it take for them to be recognised, and how many patients will die in the meantime?

1 Darrell, J H, and Garrod, L P, British Medical fournal, 1969, 2, 481.

2 Meers, P O, et al, Lancet, 1973, 2, 1189.

${ }^{3}$ Maki, D G, et al, American fournal of Medicine, 1976, 60, 471.

${ }^{4}$ Lapage, S P, Johnson, R, and Holmes, B, Lancet, 1973, 2, 284.

${ }^{5}$ Maki, D G, and Martin, W, fournal of Infectious Diseases, 1975, 131, 267.

${ }^{6}$ Maki, D G, Goldmann, D A, and Rhame, F S, Annals of Internal Medicine, 1973, 79, 867.

${ }^{7}$ Michaels, I, and Ruebner, B, Lancet, 1953, 1, 772.

\section{Welded hair}

Shampooing in moderation must be one of the least hazardous things that happens to the hair. But there is a licence for excess in the saying that "cleanliness is next to godliness," whence the aggressively virtuous can derive a rationale for their frequent and vigorous ablutions. Armed with their scallop shell of soap they pursue their hygienic pilgrimage through life, degreasing their scalps at every opportunity and subjecting them to an ordeal by water and hot air. Their activities may be unsuspected until some chance remark, such as, "I wash my hair every day, doctor, and shampoo it once a week." The opposite extreme-total disregard for the person-is more difficult for self-respecting humanity to stomach, but the unshampooed often seem blissfully ignorant of their own state even though they are anaemic from the bloodthirsty appetite of their attendant fauna, which disport themselves in the glades of their host's tangled and filthy locks.

While Agnes Savill ${ }^{1}$ cited ladies whose hair and scalp were kept immaculate with brush and comb only, for most of humanity a weekly shampoo is thought desirable. If harm results from these weekly domiciliary shampoos its victims fail to appear in the clinic. Allergies to the constituents of some shampoos, such as formaldehyde or parabens, have been described $^{2}$ but are rarely seen and are less well known than allergies to other hairdressing materials. Shampoos are more important as primary irritants than as sensitisers, and hence 